\title{
Effect of the Radial Pressure Gradient on the Secondary Flow Generated in an Annular Turbine Cascade
}

\author{
Hesham M. El-Batsh \\ Mechanical Engineering Department, Benha Faculty of Engineering, Benha University, Benha 13512, Egypt \\ Correspondence should be addressed to Hesham M. El-Batsh, helbatsh@yahoo.com
}

Received 17 October 2011; Revised 19 March 2012; Accepted 20 March 2012

Academic Editor: J.-C. Han

Copyright (C) 2012 Hesham M. El-Batsh. This is an open access article distributed under the Creative Commons Attribution License, which permits unrestricted use, distribution, and reproduction in any medium, provided the original work is properly cited.

\begin{abstract}
This paper introduces an investigation of the effect of radial pressure gradient on the secondary flow generated in turbine cascades. Laboratory measurements were performed using an annular sector cascade which allowed the investigation using relatively small number of blades. The flow was measured upstream and downstream of the cascade using a calibrated five-hole pressure probe. The three-dimensional Reynolds Averaged Navier Stokes equations were solved to understand flow physics. Turbulence was modeled using eddy-viscosity assumption and the two-equation Shear Stress Transport (SST) $k-\omega$ model. The results obtained through this study showed that the secondary flow is significantly affected by the pressure gradient along blade span. The experimental measurements and the numerical calculations predicted passage vortex near blade hub which had larger and stronger values than that predicted near blade tip. The loss distribution revealed that secondary flow loss was concentrated near blade hub. It is recommended that attempts of reducing secondary flow in annular cascade should put emphasis on the passage vortex near the hub.
\end{abstract}

\section{Introduction}

Large-scale steam and gas turbines are always used in power generation and industrial applications. Therefore, turbine efficiency and performance have major concern. The losses in a turbine can be divided into profile loss, secondary flow loss, and tip clearance loss. The profile loss is caused by the growth of the boundary layer on the blades. Secondary flow loss is generated due to the deflection through blade channel. Tip leakage loss is induced due to pressure difference between blade pressure side and blade suction side when the tip clearance gap exists. There are many factors which influence turbine losses. The pressure gradient, turbulence level, blade geometry, incoming velocity, and inlet boundary layer thickness represent important parameters affecting turbine efficiency.

It is practically very difficult to perform detailed flow field measurements in an engine at operating conditions. Understanding the physics that governs the flow and the associated turbine cascade losses has been obtained through wind tunnel experiments. These laboratory tests not only allow detailed flow field measurements but also give the experimenter the possibility to investigate the effect of several parameters separately.

Experimental studies using linear turbine cascades introduce the aspect of flow periodicity by arranging a number of blades of constant cross-sections separated by a constant pitch. Linear cascade experiments provide several advantages such as geometric simplicity, simple adjustment, large blade sections, and simply changing incidence angle. They are used as a tool to provide quasi-three-dimensional blade-to-blade data for the simulation of the flow. Linear cascades have been used extensively and have succeeded in providing a better understanding of the physics involved. They simplified the problem and allowed the usage of large-scale cascades to provide better detailed measurements in different regions of the turbine. Linear turbine cascades have been used very extensively for basic investigations of secondary flows through turbine cascades [1-8].

The reliability of the experimental data is improved by using numerical calculations for the interpretation of the data. Linear turbine cascade measurements are also 
commonly used in defining the proper boundary conditions for numerical calculations and the selection of appropriate turbulence models and validation of the numerical techniques.

Although the experimental data of the secondary flows obtained in linear cascades are very valuable for numerical validation, they cannot be used directly for calculating turbine flows where the radial static pressure gradient field plays a particularly important role with respect to the spanwise distribution of losses and outlet flow angles. Figure 1 shows proposed static pressure gradients through linear and annular cascades. In linear cascades, the static pressure changes along the blade passage producing pressure gradient from the blade pressure side to the blade suction side. This gradient induces secondary flow which is symmetrical along blade midspan in linear cascades. In actual turbines, the cascades are annular with pressure gradient along blade passage from the pressure side to the suction side and a pressure gradient along blade span caused by the curvature of the endwalls at the hub and at the casing. As a consequence, the secondary flow in actual machines is not symmetrical along blade midspan, and annular cascades measurements are required to represent the actual machines.

The annular turbine cascade consists of an annular space between two concentric cylinders which contains a turbine blade row. The main advantage of annular cascades over linear cascades is the possibility of simulating the radial static pressure gradient and, therefore, simulating turbine flow conditions more closely than the linear cascade. However, the increased number of blades in annular cascades makes the blades have smaller scale than that of linear cascades causing increased probe blockage effects and higher measurement errors due to stronger pressure gradients. Recently, El-Batsh and Bassily Hanna [9] obtained measurements using annular cascade with tip clearance gap and relatively short blades which have aspect ratio of 0.8 and at Reynolds number $9 \times 10^{4}$. Matsunuma [10] used annular cascade with blade span of $75 \mathrm{~mm}$ and blade aspect ratio of about 1 with flow Reynolds number from $4 \times 10^{4}$ to $26 \times 10^{4}$. A very comprehensive review of advanced applicable techniques to both linear and annular cascade testing has been published by Hirsch [11].

Annular large-scale sector cascades are used as a compromise between the advantages of linear and annular cascades simulating properly the radial pressure gradient field and increasing the blade size which enables accurate measurements for laboratory investigation using a relatively low number of blades. In addition, blades with large aspect ratios including blade twist and changes of section area along blade span can be examined using annular sector cascades. Furthermore, existing engine parts can be examined, and the blade profiles may be obtained from the engine. The established radial pressure gradients ensure that secondary flows develop as they would exist in an operating engine. Model and testing costs for annular sector cascades are considerably lower than those for their fully annular equivalents.

This study aims to investigate the effect of radial pressure gradient on the three-dimensional flow through radial turbine cascades without tip clearance. The study aims also to predict secondary flow generated in real turbines. This is achieved by using an annular turbine cascade sector with large-scale turbine blades. The effects of the radial pressure gradient on the secondary flow and loss mechanism through the cascade are examined. To interpret the data and to understand the flow physics, numerical calculations are performed as well.

\section{Experimental Setup}

Limited information is available concerning experiment design using annular sector cascades. Vogt and Fransson [12] successfully developed an annular sector facility for studies of the aeromechanical phenomena in axial flow turbomachines. Reducing the annular cascade to a sector cascade allowed them to maximize the size of the test object. Povey et al. [13] conducted a direct aerodynamic comparison of the flow in an annular cascade facility and in an annular sector facility of five vane passages. They obtained excellent periodicity across most of the sector. The design of the annular sector cascade presented in the present study is based on the guidelines of Povey et al. [13].

2.1. Annular Sector Turbine Cascade. The annular sector cascade constructed in this study was equipped with 5 blades representing blade profile exact replicas of the first stage rotor of the gas turbine engine from General Electric working in electric power generation. The rotor contains 92 blades and has hub and tip diameters of $1946 \mathrm{~mm}$ and $2366 \mathrm{~mm}$, respectively. The blade profile is tapered to represent actual turbine blade with different cross-sections along blade span. Radial pressure gradient exists in the rotor and in the stator. In the stator, flow deflection caused by the hub and the casing produces radial pressure gradient which changes secondary flow pattern between blade tip and blade root. In the rotor, the problem is more complicated due to the rotation of the blades and the associated centrifugal force. This effect makes the design usually based on radial equilibrium. However, experimental measurements for the rotor during rotation are rather complicated and in most cases are not possible if we have to consider the actual speed of rotation. Therefore, the measurements are performed in the present study while the blades are fixed. This would give the flow field in the absence of the centrifugal forces. The measurements also allow numerical model verification. Further study will be performed which allows blade rotation using the adopted numerical technique which would simulate the actual case in the machine with the same rotating speed. Blade coordinates were obtained using a scanning system which is used in reverse engineering. It provided the blade coordinates over eight cross-sections along blade span. Figure 2 shows the blade profile at the tip and the three-dimensional blade profile, and Table 1 summarizes cascade parameters.

The sector annular cascade was constructed from two concentric cylinder sectors representing the hub and the casing. The diameters of the cylinders are the hub and tip diameters as given in the real machine. The measurements were obtained upstream and downstream of the middle blade 


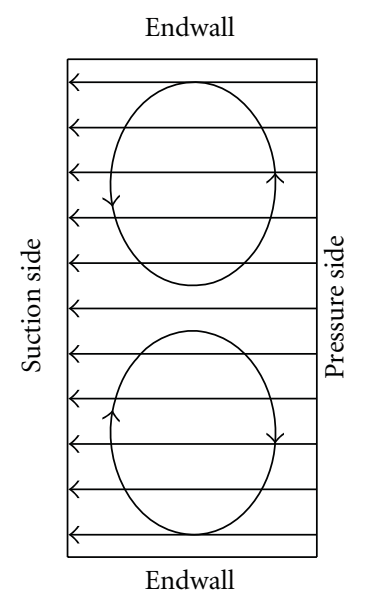

Pressure gradient in linear cascade caused by flow turning and associated secondary flow

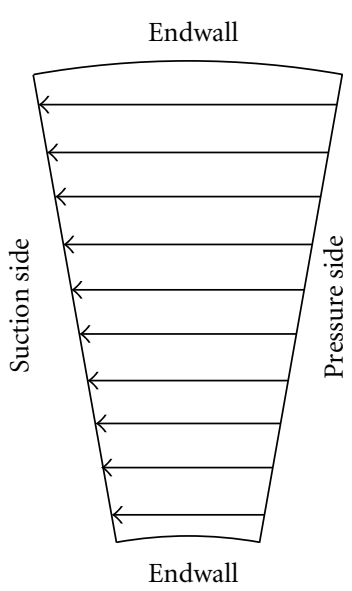

Annular cascade passage pressure gradient due to turning the flow

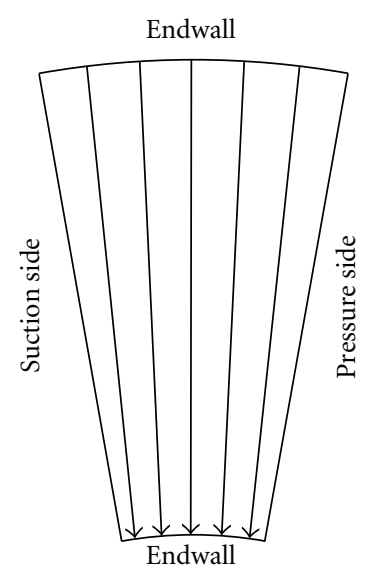

Annular cascade radial pressure gradient due to swirling flow

Figure 1: Pressure gradient in linear and in annular cascades.
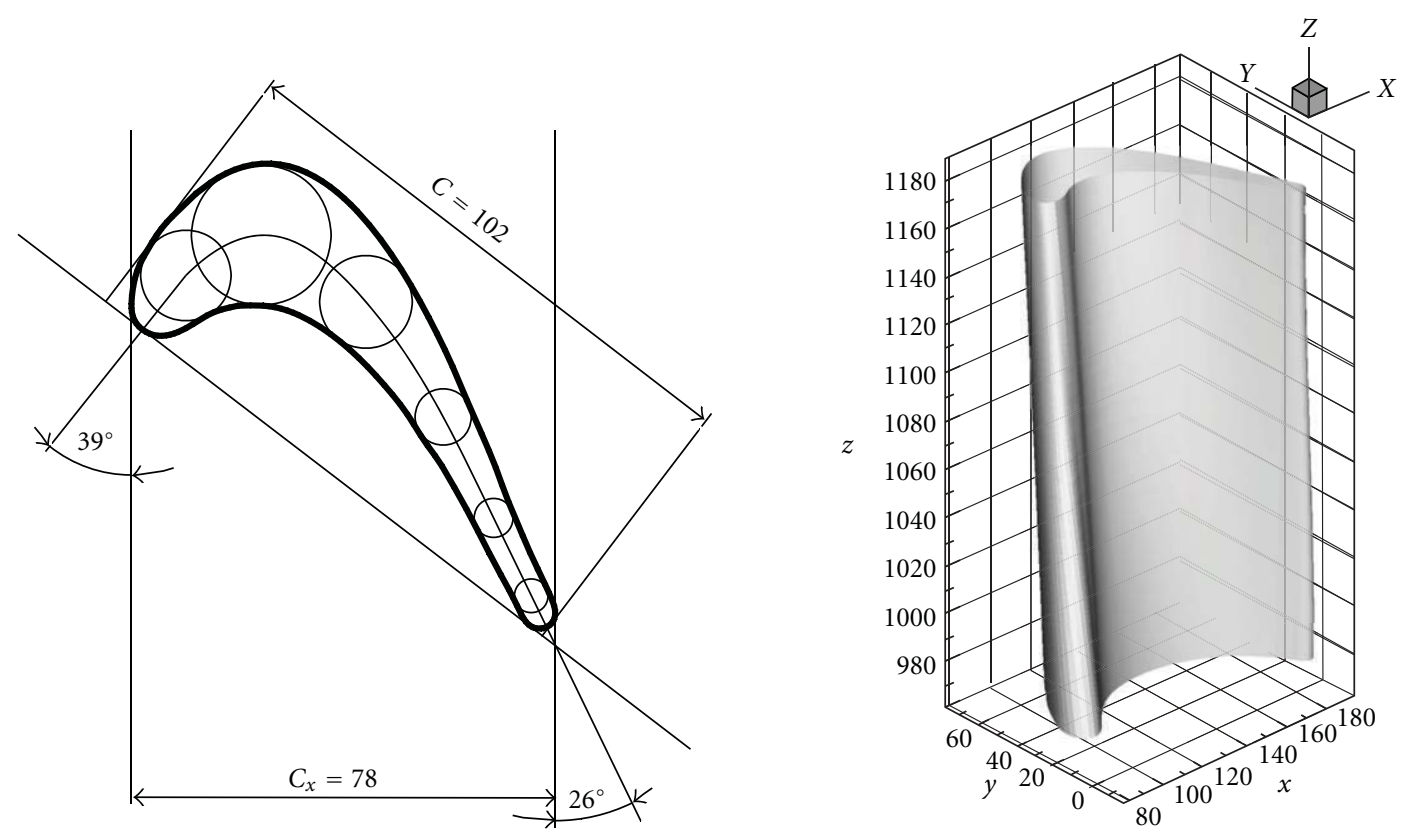

Dimensions are in $\mathrm{mm}$

FIGURE 2: Diagram illustrating cascade parameters at the tip and the three-dimensional blade profile.

which was obtained from the real gas turbine engine and was not manufactured. The other blades were manufactured by casting and finished to obtain smooth surfaces. The blades were fixed to the hub and the casing, and, therefore, there was no tip clearance gap. The angle between two adjacent blades is $360 / 92=3.913^{\circ}$.

The annular sector cascade (Figure 3 ) was examined by using a low-speed wind tunnel which is a blow-down facility. The wind tunnel is equipped with a centrifugal fan which was driven by a $10 \mathrm{HP}$ electric motor. Inlet air velocity was adjusted by using a throttling system at fan inlet. The distance between the annular sector cascade and the fan is about $4.5 \mathrm{~m}$. In order to obtain uniform flow at the inlet to the test section, three grids were used at distances of $0.89 \mathrm{~m}, 1.41 \mathrm{~m}$, and $1.93 \mathrm{~m}$.

The upstream flow measurements were obtained at a distance of $105 \mathrm{~mm}$ upstream of the blade leading edge while the downstream measurements were obtained at 
TABLE 1: Cascade parameters.

\begin{tabular}{lccc}
\hline & Root & & tip \\
\hline Chord length, $C[\mathrm{~mm}]$ & 107 & & 102 \\
Axial chord, $C_{x}[\mathrm{~mm}]$ & 105 & & 78 \\
Inlet angle, $\beta_{1}\left[^{\circ}\right]$ & & 39 & \\
Exit angle, $\beta_{2}\left[^{\circ}\right]$ & & 26 & \\
Blade span, $h[\mathrm{~mm}]$ & & 210 & \\
Blade spacing $(\mathrm{pitch}), S[\mathrm{~mm}]$ & 66.45 & & 80.8 \\
Aspect ratio, $h / C[-]$ & 1.96 & & 2.06 \\
Inner diameter, $D_{H}[\mathrm{~mm}]$ & & 1946 & \\
Outer diameter, $D_{T}[\mathrm{~mm}]$ & & 2366 & \\
Number of blades, $N[-]$ & & 92 & \\
\hline
\end{tabular}

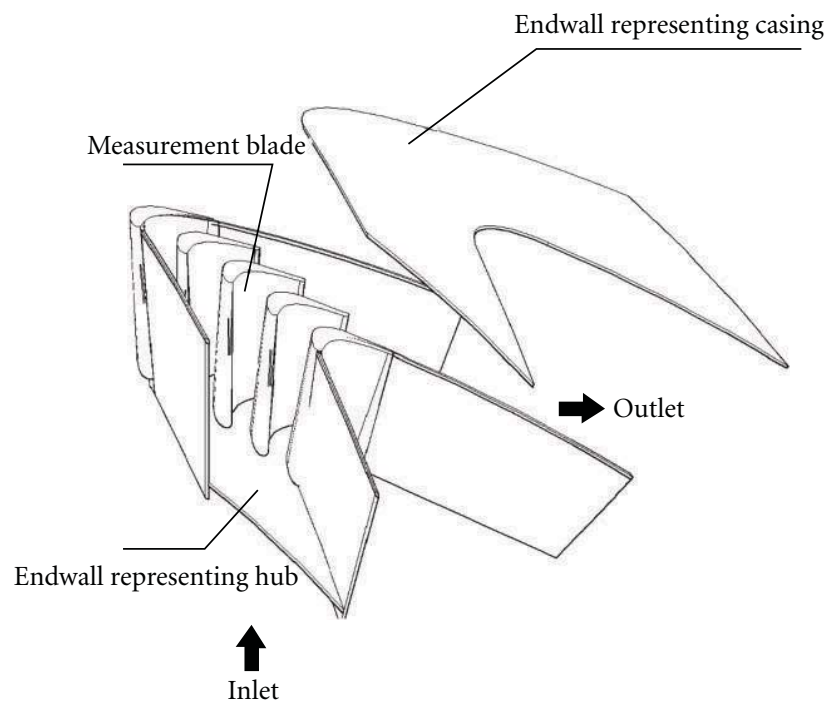

Figure 3: Annular sector cascade.

a distance of $52.5 \mathrm{~mm}$ downstream of the blade trailing edge. The flow was measured downstream of the annular sector cascade using a mesh with 8 equally spaced points in the pitchwise direction and 25 points in the spanwise direction. A traverse mechanism was used to allow probe rotation in the pitchwise direction and translation in the spanwise direction. The upstream and the downstream measurement meshes were vertical. The probe was moved with a step of $5 \mathrm{~mm}$ in the region close to the hub and to the casing and $10 \mathrm{~mm}$ elsewhere. Figure 4 shows the measurement mesh with a total number of 200 grid points.

2.2. Measuring Instrumentations. Five-hole pressure probes are widely used in turbomachinery for the measurement of fluid flow because they are relatively accurate and robust. Although more advanced techniques are available today such as laser and hot wire, the advantages of the five-hole probe make it superior for turbomachinery flow measurements. They are recently used for turbomachinery applications by many researchers $[8-10,14,15]$.

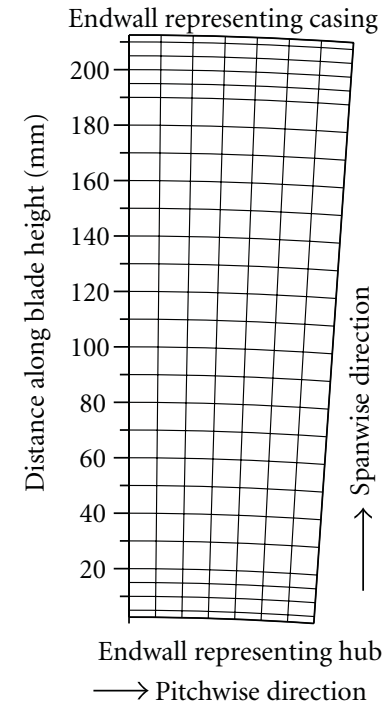

FIgURE 4: Measurements mesh.

The five-hole probes can be used to measure total and static pressures as well as gas velocity and flow angle. This is achieved by extensive calibrations for the probe to cover all of the expected flow conditions that will be encountered by the probe during wind tunnel measurements. A five-hole probe has five pressure ports which are distributed on a conical tip. The five-hole probe used in this study has L-shape with tip diameter of $3 \mathrm{~mm}$ as shown in Figure 5(a). One-hole port is located at the apex of the cone, and the other hole ports $2-5$ are uniformly distributed around the central port. This probe geometry provides the capability of making accurate measurements for flow angles of up to $\pm 30^{\circ}$ if the probe has been properly calibrated within those limits. The threedimensional velocity vector that is measured with this probe is expressed in the probe axis coordinate system shown in Figure 5(b). Typically the incidence angle of the flow with respect to the probe tip is defined using pitch angle $\alpha$ and yaw angle $\beta$. Hence, the velocity components in the probe axis coordinate system may be expressed using these angles as well as the velocity magnitude.

2.3. Calibration Procedure. Probe calibration was performed here by placing the probe into a calibration rig with uniform and one-dimensional flow. The total pressure $\left(P_{t}\right)$, static pressure $\left(P_{s}\right)$, and the orientation of the probe tip with respect to the flow field direction represented by pitch and yaw angles are known. The pressures at the five ports of the probe tip $\left(p_{1}, p_{2}, p_{3}, p_{4}, p_{5}\right)$ were measured. The probe was rotated in the pitch and yaw directions using a traversing mechanism to allow measuring pressure signals obtained from the five holes. The probe was rotated at a set of angle combinations pitch and yaw that cover the range of incidence angles that are expected for the probe to encounter during the wind tunnel measurements. The calibration was performed in the range for pitch and yaw angles from -30 to +30 . The pressure signals obtained from the probe are measured using digital micromanometers with full scale 


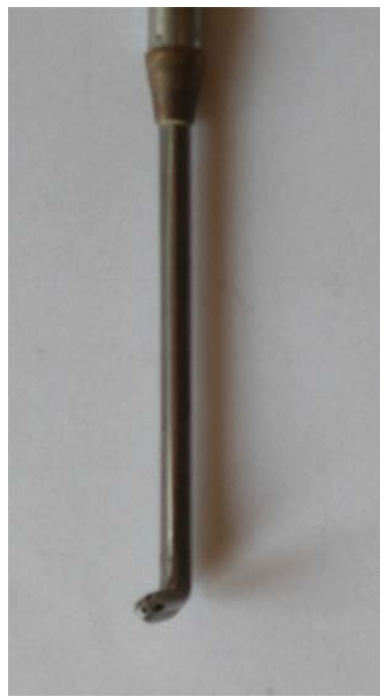

(a) Photo for the five-hole probe

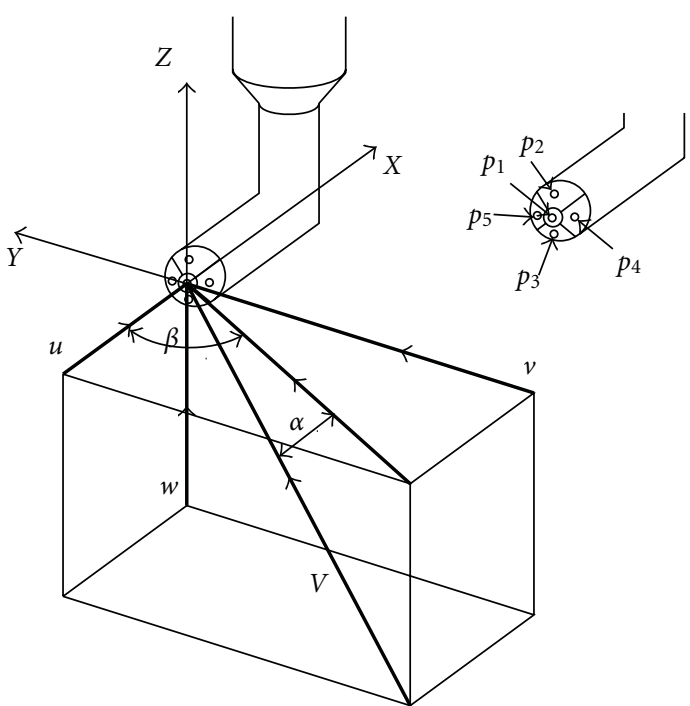

(b) Probe coordinate system

FIGURE 5: L-shaped five-hole probe with a conical tip.

of $3700 \mathrm{~Pa}$ and accuracy of $0.1 \%$. The pressure data were expressed as a group of non-dimensional coefficients as follows.

The pitch angle coefficient

$$
k_{\alpha}=\frac{\left(p_{2}-p_{3}\right)}{p_{1}-\bar{p}},
$$

the yaw angle coefficient

$$
k_{\beta}=\frac{\left(p_{4}-p_{5}\right)}{p_{1}-\bar{p}}
$$

total pressure coefficient

$$
k_{t}=\frac{\left(p_{1}-P_{t}\right)}{p_{1}-\bar{p}}
$$

the static pressure coefficient

$$
\begin{gathered}
k_{s}=\frac{\left(p_{1}-p_{s}\right)}{p_{1}-\bar{p}}, \\
\bar{p}=\frac{1}{4}\left(p_{2}+p_{3}+p_{4}+p_{5}\right) .
\end{gathered}
$$

2.4. Measurement Procedure. During wind tunnel measurements, the probe was inserted into the unknown flow field, and the pressures at the tip of the probe $\left(p_{1}, p_{2}, p_{3}, p_{4}, p_{5}\right)$ were measured for each test point. The five pressures were used to calculate the pitch and yaw angle coefficients $\left(k_{\alpha}\right.$ and $\left.k_{\beta}\right)$. These values were used to obtain flow angles $\alpha$ and $\beta$ using calibration map of Figure 6. Then pitch angle and yaw angle are used in combination with the calibration maps (Figures 7 and 8) to obtain the total and static pressure coefficients $\left(k_{t}, k_{s}\right)$. Rearranging the equations defining the total and static pressure coefficients, the velocity magnitude is given by

$$
|V|=\sqrt{\left(\frac{2}{\rho}\right)\left(p_{1}-\bar{p}\right)\left(1+k_{s}-k_{t}\right)} .
$$

The velocity components were obtained as

$$
\begin{gathered}
u=|V| \cos (\alpha) \cos (\beta), \\
v=|V| \sin (\alpha), \\
w=|V| \cos (\alpha) \sin (\beta) .
\end{gathered}
$$

The uncertainties of the calculated results were estimated on the basis of the uncertainties in the primary measured values. The result $R$ is given function of the independent variables $x_{1}, x_{2}, x_{3}, \ldots, x_{n}$. Thus,

$$
R=R\left(x_{1}, x_{2}, x_{3}, \ldots, x_{n}\right) .
$$

Let $w_{R}$ be the uncertainty of the result and $w_{1}, w_{2}, w_{3}, \ldots, w_{n}$ be the uncertainties in the independent variables. If the uncertainties in the independent variables are all given, then, the uncertainties in the results are given by

$$
w_{R}=\left[\left(\frac{\partial R}{\partial x_{1}} w_{1}\right)^{2}+\left(\frac{\partial R}{\partial x_{2}} w_{2}\right)^{2}+\cdots+\left(\frac{\partial R}{\partial x_{n}} w_{n}\right)^{2}\right]^{1 / 2} .
$$

From the experimental measurements, the uncertainty in the velocity, flow angle, and total pressure was found to be within $3 \%, 5 \%$, and $9 \%$, respectively.

\section{Numerical Predictions}

The three-dimensional flow through the cascade was obtained by solving the flow governing equations. In all 


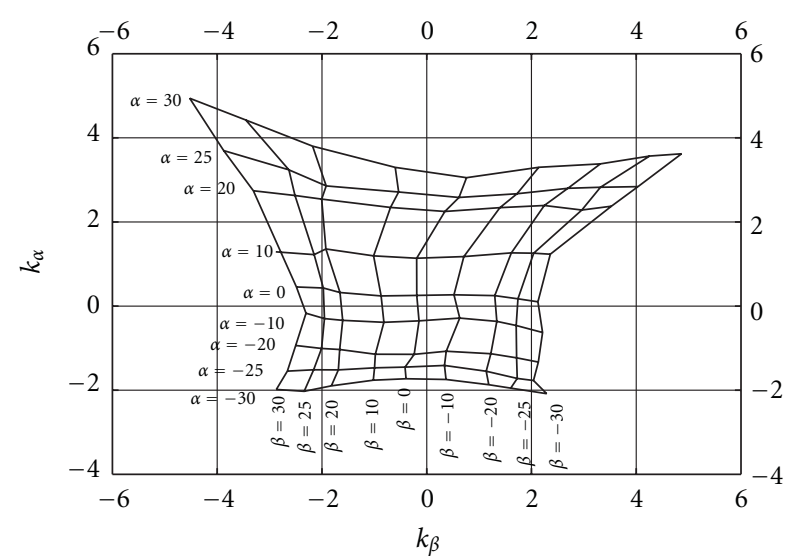

Figure 6: Calibration map for the yaw and pitch dimensionless coefficients.

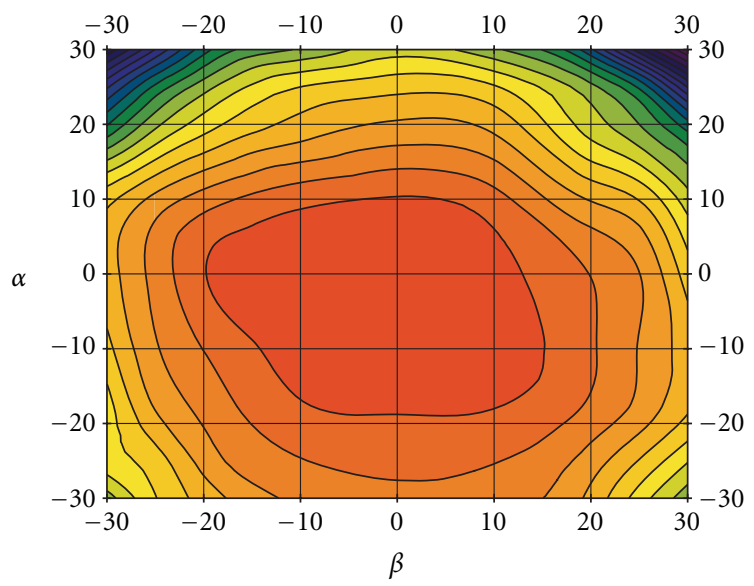

IIIIIIIIIIIIIIIIII

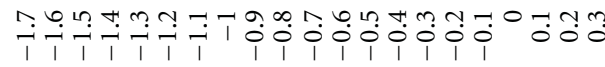
$k_{t}$

Figure 7: Calibration map for the total pressure coefficient.

calculations performed here, the Mach number was small, and therefore the flow was considered incompressible, and as a consequence solving energy equation was not required. Since the flow through turbine cascades is almost turbulent, an appropriate turbulence model was required. The selected turbulence model is able to predict the losses with reasonable accuracy. A commercial CFD code was employed to solve the flow-governing equations.

3.1. Governing Equations. Fluid flow characteristics are described by the conservation of mass (continuity equation) and momentum (Navier-Stokes equations). For turbulent flows, Reynolds averaging procedure is commonly used, and the governing equations are called Reynolds Averaged
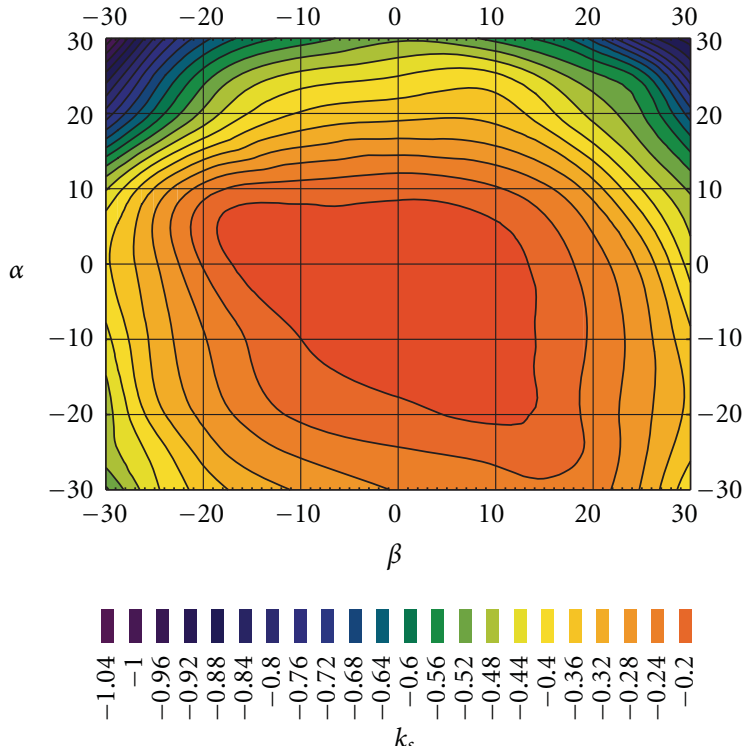

Figure 8: Calibration map for the static pressure coefficient.

Navier Stokes equations. For incompressible turbulent flow neglecting external forces they are given by [16]

$$
\begin{gathered}
\frac{\partial \overline{u_{i}}}{\partial x_{i}}=0 \\
\rho \frac{\partial}{\partial x_{j}}\left(\overline{u_{i} u_{j}}\right)=-\frac{\partial P}{\partial x_{i}}+\frac{\partial}{\partial x_{j}}\left[\mu\left(\frac{\partial \overline{u_{i}}}{\partial x_{j}}+\frac{\partial \overline{u_{j}}}{\partial x_{i}}\right)-\rho \overline{u_{i}^{\prime} u_{j}^{\prime}}\right],
\end{gathered}
$$

where the velocities $\overline{u_{i}}$ are mean values, $u_{i}^{\prime}$ are the fluctuating values, and $-\rho \overline{u_{i}^{\prime} u_{j}^{\prime}}$ are the Reynolds stresses which are calculated using eddy viscosity turbulence models as

$$
-\rho \overline{u_{i}^{\prime} u_{j}^{\prime}}=\mu_{t}\left(\frac{\partial \overline{u_{i}}}{\partial x_{j}}+\frac{\partial \overline{u_{j}}}{\partial x_{i}}\right)-\frac{2}{3} \rho k \delta_{i j} .
$$

The eddy or turbulent viscosity $\mu_{t}$ was calculated in this study using the Shear Stress Transport (SST) $k-\omega$ model; $\delta_{i j}$ is the Kronecker second-order tensor given by

$$
\delta_{i j}= \begin{cases}1 & \text { if } i=j, \\ 0 & \text { if } i \neq j .\end{cases}
$$

3.2. The SST k- $\omega$ Model. Bardina et al. [17] discussed the performance of different turbulence models. They found that the SST $k-\omega$ model can predict the flows with strong adverse pressure gradients and separation. The SST $k-\omega$ model was also recently used successfully to predict the threedimensional complex flow through axial flow turbine blades $[9,18]$.

The SST $k-\omega$ model is an empirical model based on model transport equations for the turbulence kinetic energy $k$ and the specific dissipation rate $\omega$. Eddy or turbulent viscosity is calculated as

$$
\mu_{t}=\frac{\rho k / \omega}{\max \left(1 ; \Omega F_{2} /\left(a_{1} \omega\right)\right)} .
$$




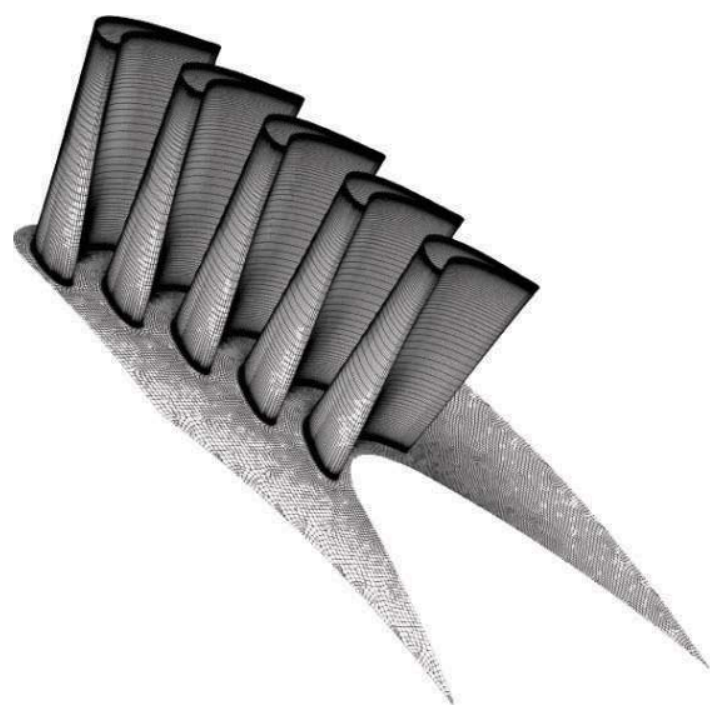

(a)

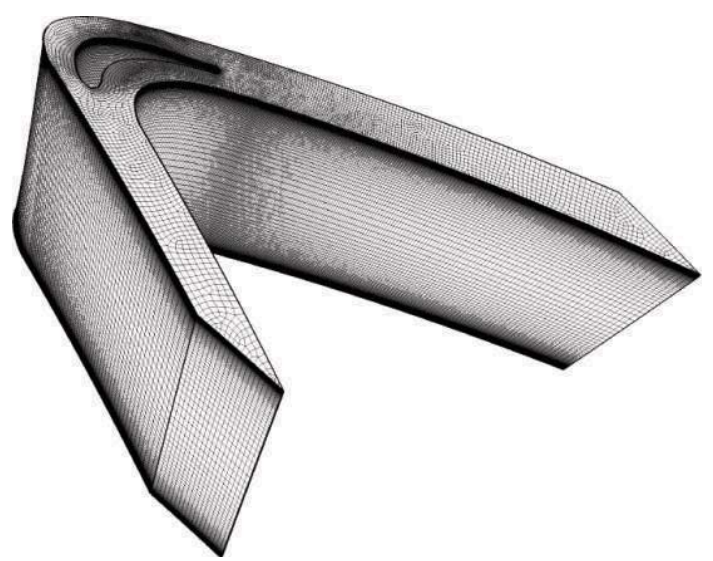

(b)

FIgURE 9: Computational grid.

In the turbulent boundary layers, the maximum value of the eddy viscosity is limited by forcing the turbulent shear stress to be bounded by the turbulent kinetic energy time $a_{1}$. This effect is achieved with the auxiliary function $F_{2}$ and the absolute value of vorticity $\Omega$. The auxiliary function $F_{2}$ is defined as a function of wall distance $y$ as

$$
F_{2}=\tanh \left\{\left[\max \left(2 \frac{\sqrt{k}}{0.09 \omega y} ; \frac{500 \mu}{\rho y^{2} \omega}\right)\right]^{2}\right\} .
$$

The transport equations as developed by Menter [19] and presented by Bardina et al. [17] are

$$
\begin{gathered}
\frac{\partial}{\partial x_{j}}\left[\rho k \overline{u_{j}}-\left(\mu+\sigma_{k} \mu_{t}\right) \frac{\partial k}{\partial x_{j}}\right]=\tau_{i j} S_{i j}-\beta^{*} \rho \omega k, \\
\frac{\partial}{\partial x_{j}}\left[\rho \omega \overline{u_{j}}-\left(\mu+\sigma_{\omega} \mu_{t}\right) \frac{\partial \omega}{\partial x_{j}}\right] \\
=P_{\omega}-\beta^{\prime} \rho \omega^{2}+2\left(1-F_{1}\right) \frac{\rho \sigma_{\omega 2}}{\omega} \frac{\partial k}{\partial x_{j}} \frac{\partial \omega}{\partial x_{j}} .
\end{gathered}
$$

$P_{\omega}$ is the production term of $\omega$, and the function $F_{1}$ is designed to blend the model coefficient in the boundary layer zones. The constants of the model are $a_{1}$ and $\beta^{*}$. Model coefficients are $\beta^{\prime}, \gamma^{\prime}, \sigma_{k}$, and $\sigma_{\omega}$. Complete details on the model can be obtained from the original paper [19].

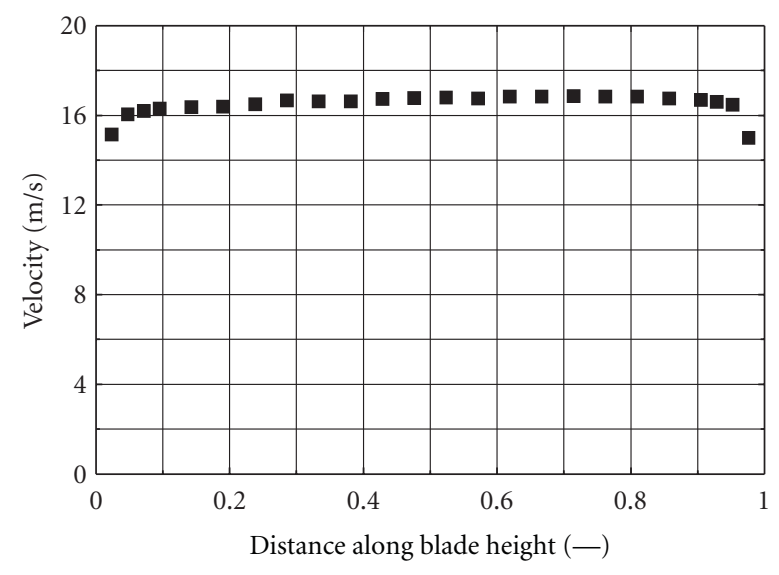

FIGURE 10: Inlet velocity distribution.

3.3. Numerical Methodology. A control-volume technique was employed to convert the differential equations to algebraic equations which can be solved numerically. The upwind scheme was used to represent the convection terms of the governing equations. The semi-implicit method for pressure-linked equation (SIMPLE) was used to solve the discretized equations. A CFD commercial code was employed here to solve the equations. All computations have been carried out using a personal computer with a single processor of Intel I5 with frequency 2.4.

3.4. Grid Generation. The flow was solved around a single blade considering periodic flow. The inlet boundary was considered at a distance of axial chord upstream of the blade leading edge while the outlet boundary was considered at a distance of axial chord downstream of the blade trailing edge. The grid was generated for the entire blade span since 


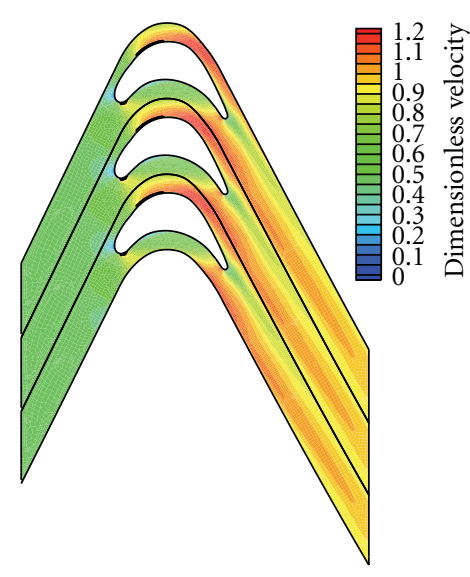

0.1 span

(a)

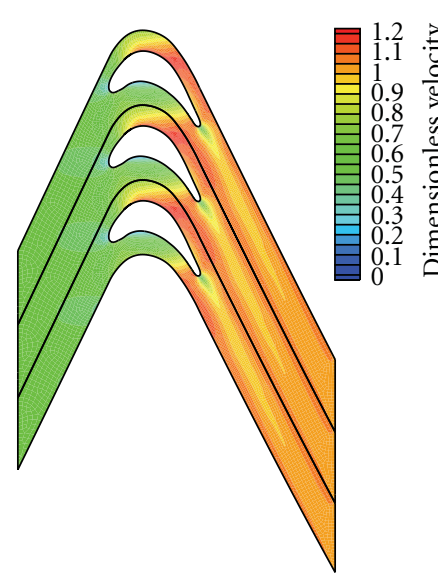

Mid-span

(b)

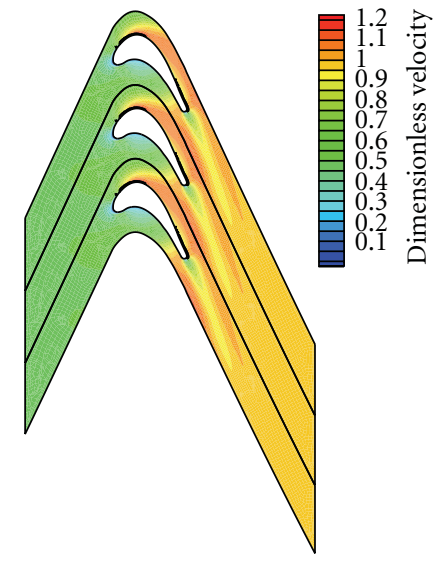

0.9 span

(c)

FIgURE 11: Dimensionless velocity in blade channel at different locations along blade span.

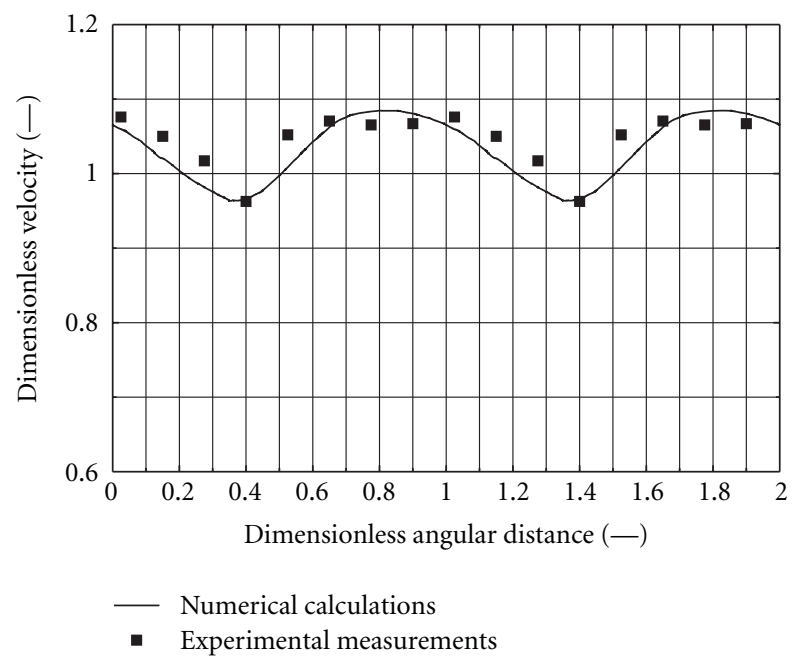

Figure 12: Dimensionless velocity on the downstream plane at blade midspan.

there is no symmetry plane at midspan which exists in linear cascades. Fixed wall boundaries were considered at the blade surface and at the hub and at the casing. A twodimensional grid was generated at the hub and was copied in the spanwise direction to form the three-dimensional grid. The grid has a total number of about 996000 grid points. The grid size was based on the grid-independent results obtained by Hildebrandt and Fottner [20] with reasonably fine mesh with 303000 grid points for the solution in linear cascade with nearly the same aspect ratio using half-span simulation. The grid was generated using multiblock topology which leads to a numerical grid of high quality expressed in terms of orthogonality and smoothness. These are essential prerequisites for obtaining accurate results in regions that are dominated by the viscous effects. Special consideration was paid during grid generation to obtain dense mesh near the blade and near the hub and tip wall. This was required

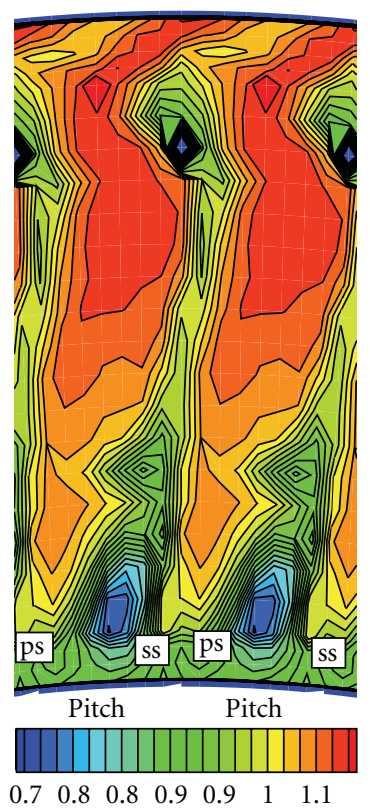

(a) Experimental measurements

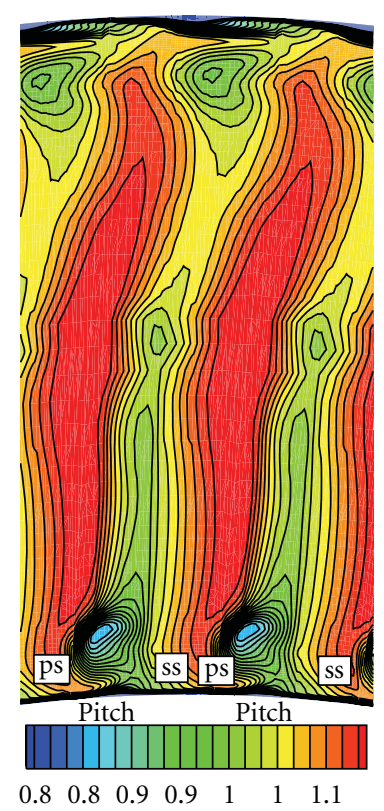

(b) Numerical calculations
Figure 13: Counter plots for the dimensionless velocity downstream the cascade.

to solve the boundary layer near the wall and to satisfy the requirements of the solution technique near the fixed walls. Figure 9 shows the computational grid.

3.5. Boundary Conditions. In the present study, the inlet velocity boundary condition was defined at the inlet while the outlet boundary condition was considered at the exit. Since the inlet velocity profile is important for the development of the secondary flow field, the inlet velocity was measured upstream of the cascade at a distance of axial chord. The velocity was measured by using the calibrated 


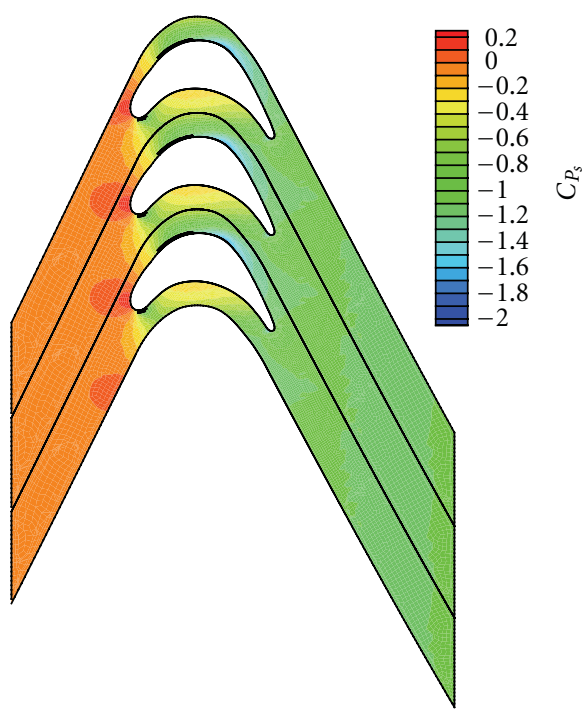

0.1 span

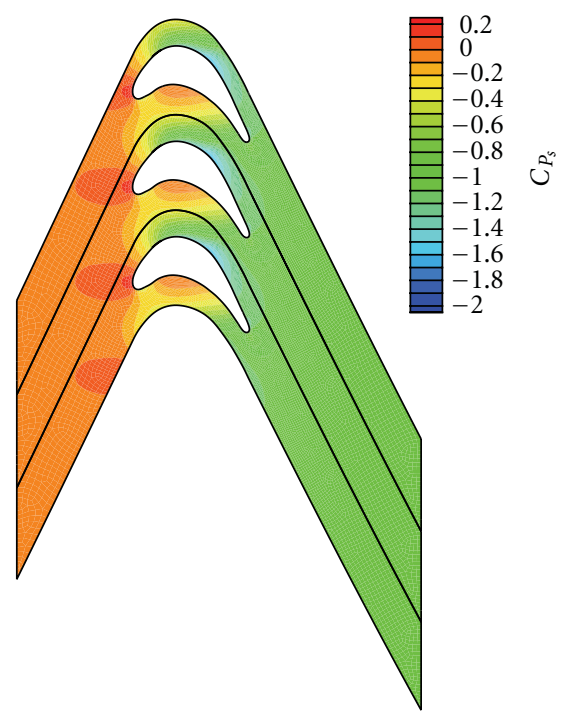

Midspan

(a)

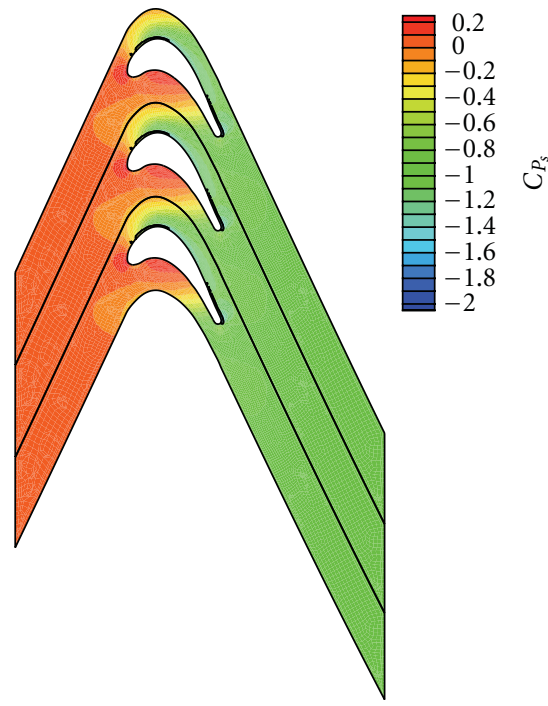

0.9 span

FIGURE 14: Static pressure coefficient through blade channel at different locations.

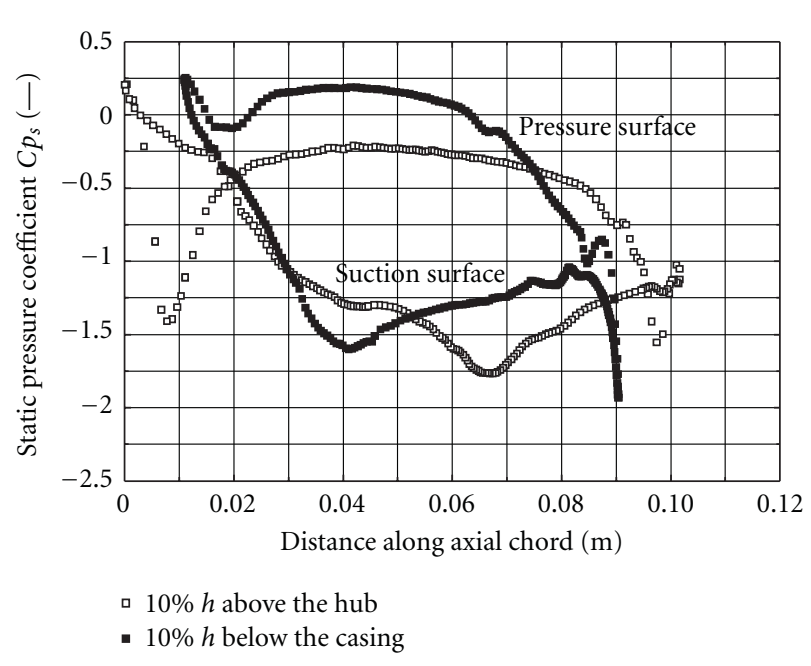

Figure 15: Static pressure coefficient calculated at two locations through blade span.

five-hole probe along a line from the hub to tip using the same spanwise distance given in Figure 4. Figure 10 shows the inlet velocity distribution used at the inlet to the numerical calculations. Matsunuma [10] studied the effect of free-stream turbulence on the flow with and without tip clearance gap. He found that the turbulence level does not significantly affect the flow field and loss behavior either with or without tip clearance gap. Inlet turbulence parameters, namely, turbulence intensity and length scale were estimated according to the general guideline for CFD calculations [21]. The inlet turbulence level was estimated in the calculations performed here as $1 \%$ while the inlet turbulence length scale was estimated based on blade span $h$ as $0.07 h$.

\section{Results and Discussion}

The results presented here can be divided into three groups. Firstly, the velocity distribution through the cascade was examined. Then the static pressure was investigated because it affects the secondary flow through the cascade. Finally, loss distribution was examined, and the locations of high losses are distinguished.

4.1. Velocity Distribution. Figure 11 shows contour plots for the dimensionless velocity calculated at three different locations along blade span. These locations exist at $10 \%$ blade span near the hub, at the midspan, and at $90 \%$ blade span near the casing. The dimensionless velocity is defined as the local velocity normalized using the mass averaged exit velocity which is given by

$$
\overline{V_{2}}=\frac{\int \dot{m} \cdot V_{2}}{\int \dot{m}}
$$

The figure indicates that at the three studied locations, the flow accelerates through the blade channel reaching the maximum velocity at the throat. On the blade suction surface, the flow accelerates to the maximum velocity and then the flow decelerates. However, on the blade pressure surface, the flow accelerates from the blade leading edge to the blade trailing edge.

Figure 12 shows the comparison between the experimentally measured and the numerically calculated velocity on the downstream plane at blade midspan. Relatively good agreement was obtained between measurements and calculations. Figure 13 shows contour plots for the experimentally measured and the numerically calculated dimensionless velocity on the downstream plane. Two passage vortices can be distinguished. The velocity level of the passage vortex 


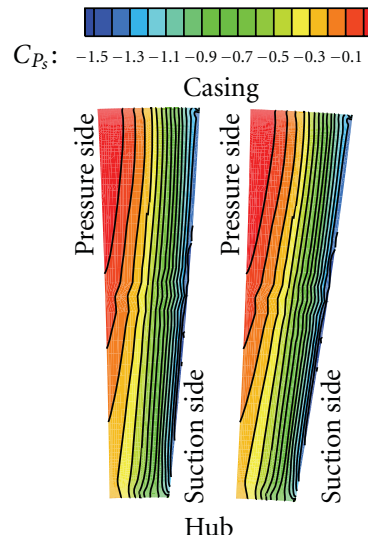

(a) Pressure distribution

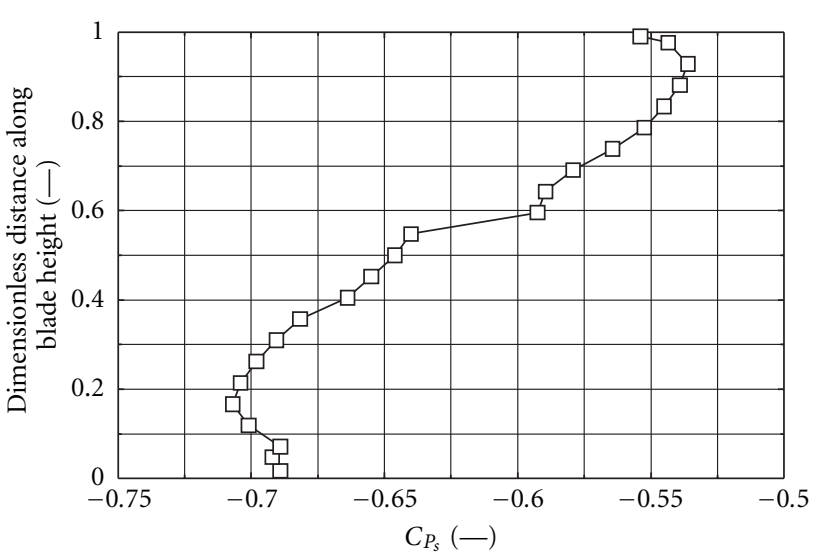

$\neg-$ Numerical calculations

(b) Pitchwise mass-averaged pressure distribution

FIGURE 16: Numerically calculated pressure distribution through blade channel $x / C_{x}=0.5$.

close to the hub is smaller than the velocity level for the passage vortex that is close to the casing. This was observed both in the numerical calculations and in the experimental measurements.

4.2. Static Pressure. The static pressure was examined through the blade channel using the static pressure coefficient which was defined as

$$
C_{P_{S}}=\frac{\left(P-P_{1}\right)}{0.5 \rho{\overline{V_{2}}}^{2}}
$$

where $P_{1}$ is the inlet static pressure, $P$ is the local static pressure, and $\rho$ is the air density.

Figure 14 shows contour plots for the distribution of the static pressure coefficient through blade channel at different levels along blade span. Generally, the figure shows nonuniform pressure distribution at the planes considered here. The figure shows that at all levels, the pressure increases at the blade pressure side and reduces at the blade suction side. The pressure calculated at the blade pressure side near the tip region at $90 \%$ span is higher than the pressure calculated at the blade midspan and at blade root. Figure 15 shows the pressure distribution on the blade surface at two locations at 0.1 and 0.9 blade span. For clarity in presentation, the blade axial chord at the tip is smaller than the blade axial chord at the hub because the studied blade is tapered blade. The figure indicated that at the same distance through the axial chord, the static pressure on the blade pressure surface is higher in the blade tip than in the blade root. The separation near the hub was caused by the constant flow angle at the inlet and the strong flow turning from inlet to exit. Figure 16(a) shows contour plot for the static pressure distribution through blade channel at a distance of midaxial chord $\left(x / C_{x}=0.5\right)$ measured from the blade leading edge. Figure 16(b) shows the variation of the pitchwise mass-averaged static pressure through blade span. The figure shows nonuniform pressure distribution

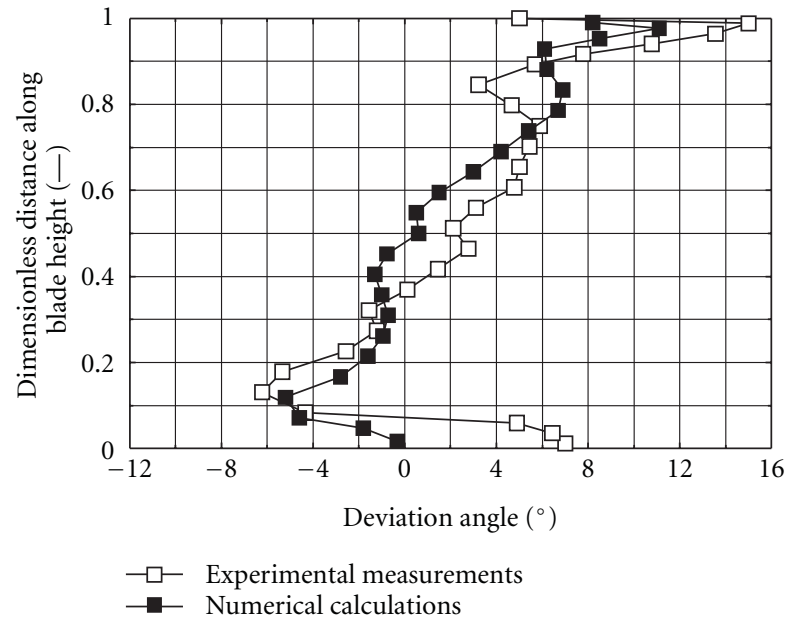

FIgURE 17: Pitchwise mass-averaged deviation angle.

along blade span. The maximum pressure was predicted at the casing on the blade pressure surface.

4.3. Flow Angle. The flow was investigated downstream of the cascade using deviation angle which was defined as

$$
\delta=\beta_{2}^{\prime}-\beta_{2},
$$

where $\beta_{2}^{\prime}$ is the exit flow angle and $\beta_{2}$ is the exit blade angle measured from the tangential direction.

Figure 17 shows the pitchwise mass averaged exit deviation angle along blade span. The deviation between measurements and calculations is about $1-2^{\circ}$ which could be attributed to the initial setting of the zero position of the probe during calibration and during cascade measurements. Nevertheless, both experimental measurements and numerical calculations give the same trend. At blade mid-span, a positive deviation angle was obtained which indicates that the flow angle is larger than the blade angle and the flow 


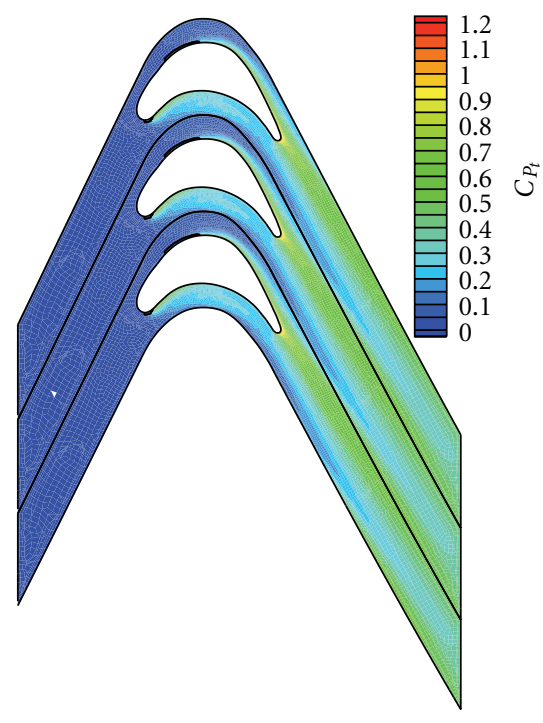

0.1 span

(a)

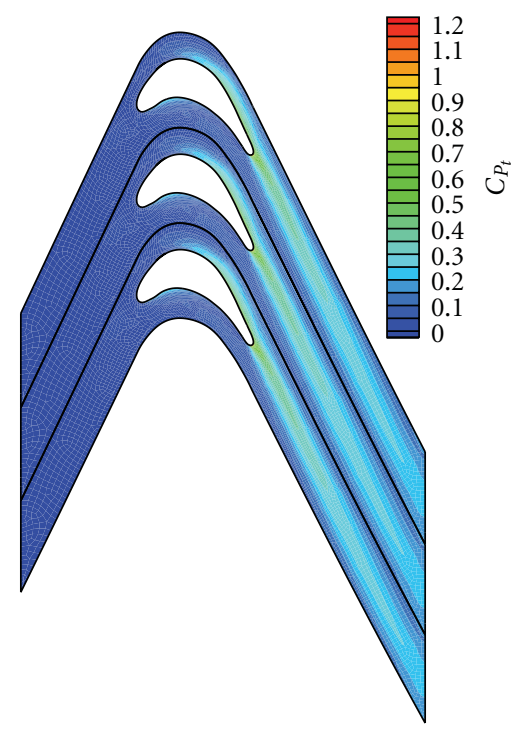

Midspan

(b)

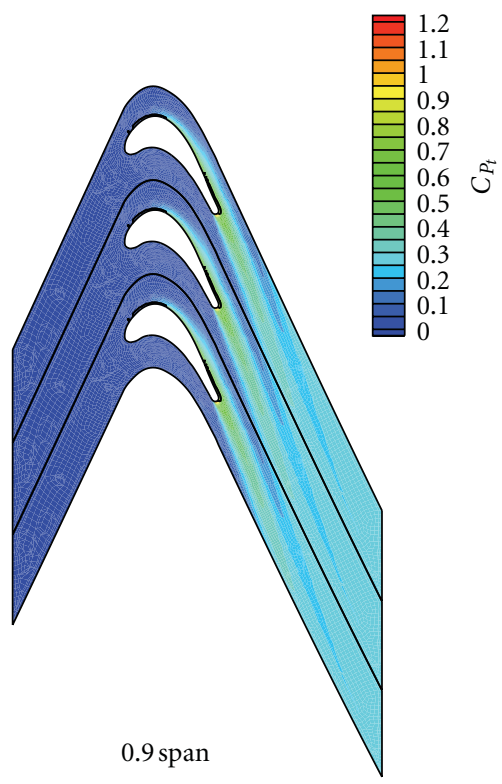

(c)

FIgURE 18: Total pressure loss coefficient through blade channel at different locations.

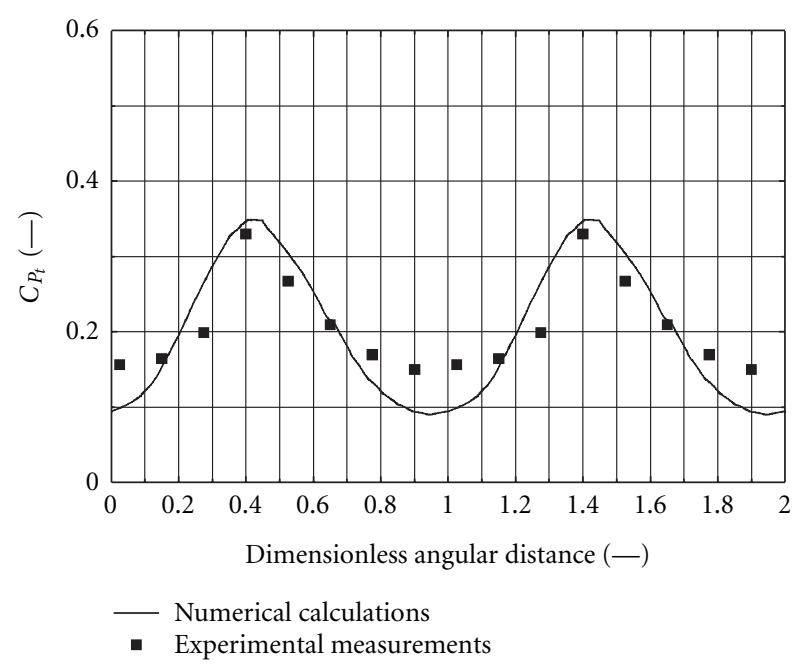

Figure 19: Total pressure loss coefficient on the downstream plane at blade midspan.

deflects from the blade pressure side to the blade suction side. This is caused by the pressure difference between blade surfaces. The figure shows also that the deviation angle increases further in the region above blade mid-span. This is caused by the increase in pressure difference between blade surfaces with blade span as demonstrated in Figure 16. The deviation angle significantly decreased due to the passage vortex near the hub and near the casing. The effect near the hub is more significant which indicates that the passage vortex near the hub is stronger than that near the casing and the pressure difference between blade surfaces is reduced.

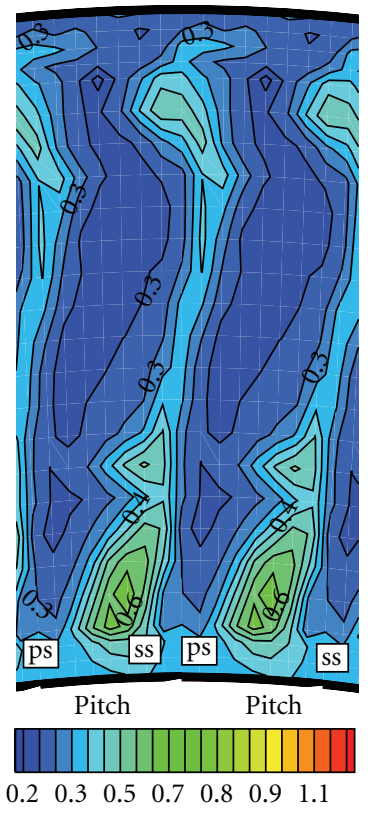

(a) Experimental measurements

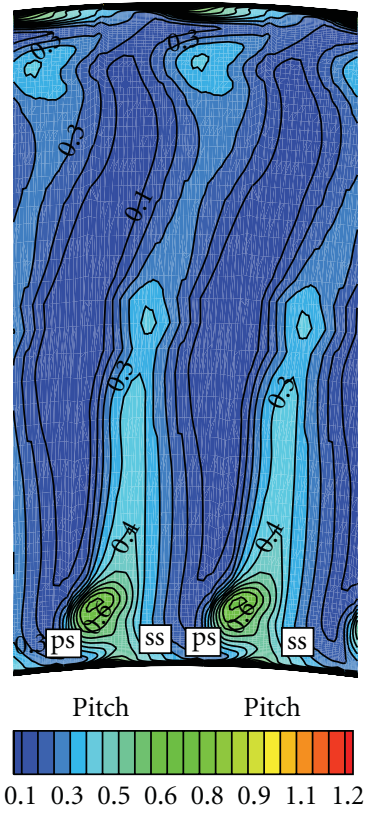

(b) Numerical calculations
Figure 20: Total pressure loss coefficient downstream the cascade.

4.4. Cascade Losses. The losses through turbine cascade were examined by using the total pressure loss coefficient which was defined as

$$
C_{P_{t}}=\frac{\left(P_{t 1}-P_{t}\right)}{0.5 \rho{\overline{V_{2}}}^{2}},
$$




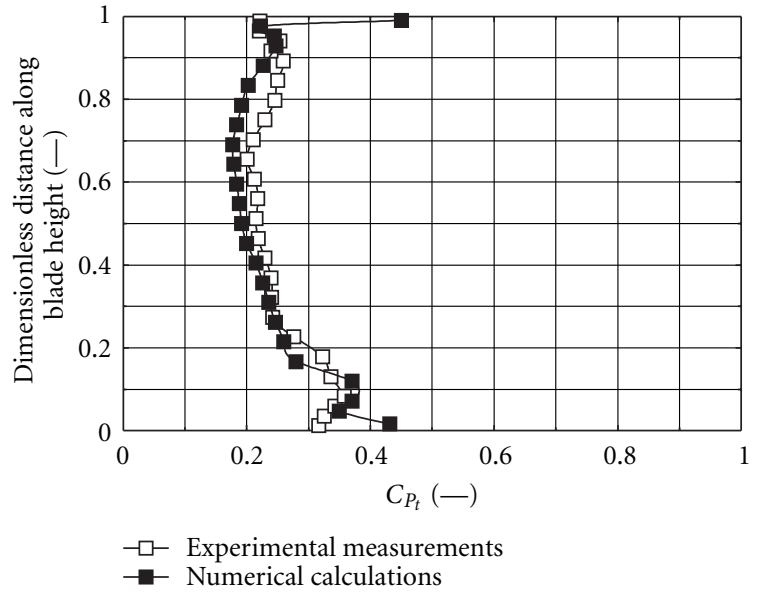

FIgURe 21: Total pressure loss coefficient along blade span.

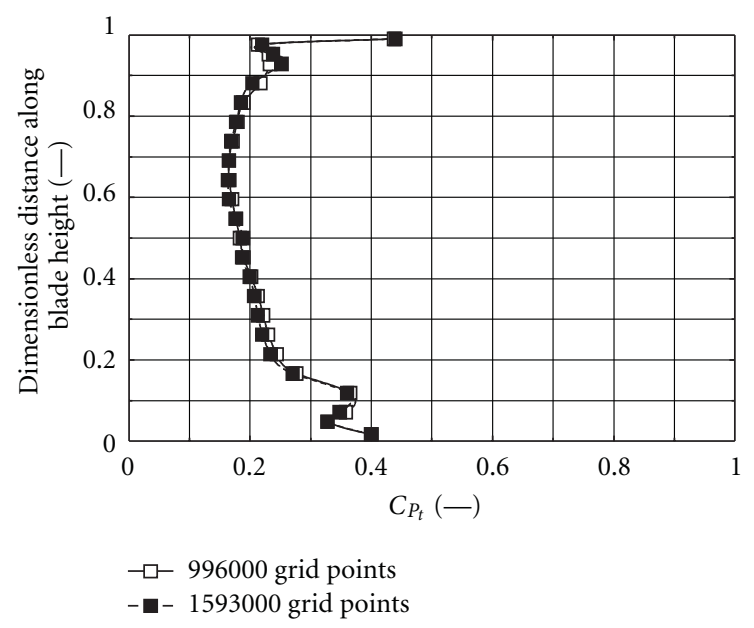

Figure 22: Effect of grid size on loss prediction.

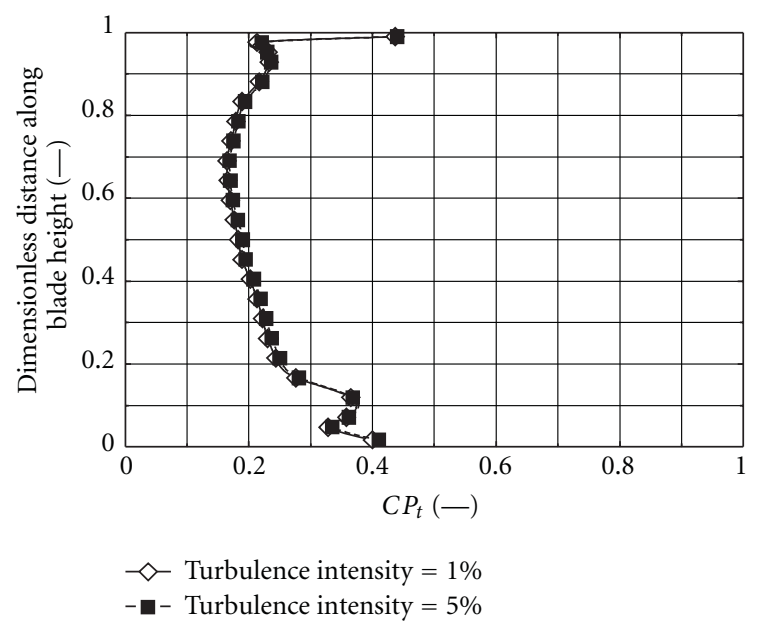

FIGURE 23: Effect of inlet turbulence intensity on loss prediction. where $P_{t 1}$ is the inlet total pressure and $P_{t}$ is the local total pressure.

Figure 18 shows contour plots for the calculated total pressure loss coefficient $C_{P_{t}}$ around the blade in the three studied planes through blade span. At blade mid-span, the losses start to increase on the blade suction surface downstream of the location of maximum velocity. Decreasing the flow velocity increases the adverse pressure and increases the boundary layer thickness. The losses at the other locations are not identical indicating that the losses were not symmetrical along blade mid-span. The losses were concentrated near the hub while the loss level near blade casing was smaller.

Figure 19 shows the comparison between the experimentally measured and the numerically calculated total pressure loss coefficient at mid-span on the downstream plane and shows relatively good agreement. Figure 20 shows contour plots for the experimental measurements and the numerical calculations of the total pressure loss coefficient on the downstream plane. Good agreement was obtained between calculations and measurements. Two passage vortices were calculated and predicted. The first passage vortex was predicted near the casing while the second passage vortex was predicted near the hub. The passage vortex near the hub was predicted by the numerical calculations and measured larger and with higher loss level than that predicted near the casing.

Figure 21 shows the pitchwise mass averaged total pressure loss coefficient through blade span. Very good agreement was obtained between experimental measurements and numerical calculations. The discrepancy near the endwall was caused by the blockage effect of the probe during the experimental measurements and the high velocity gradient in the boundary layer. The figure indicates high losses near the hub with reduced loss level near the casing. The passage vortex increased the loss level at 10\% and 90\% blade span. The measured and the calculated mass averaged loss losses were higher near the hub than that near the casing.

In order to verify the grid-independent numerical solution, a denser mesh was generated with increased grid size by $60 \%$ to obtain as mesh with 1593000 grid points. The cells were mainly added in the high gradient flow regions. Figure 22 shows the results of the grid-independent analysis by comparing the spanwise distribution of the total pressure loss coefficient using two meshes. The figure indicates that there is no significant improvement by increasing the grid size, and the solution obtained is grid independent. The uncertainty of the assumption of the inlet turbulence intensity was also examined by repeating the calculations assuming inlet turbulence intensity as $5 \%$. Figure 23 shows the comparison between the numerical results obtained assuming different inlet turbulence level. The results confirm the findings of Matsunuma [10] that secondary flow losses are independent of the inlet turbulence intensity. Figure 24 shows the secondary flow velocity vectors on the downstream plane. The figure indicates the secondary flow movements from the pressure side to the suction side near the endwall and from the suction side to the pressure side at the midspan. 


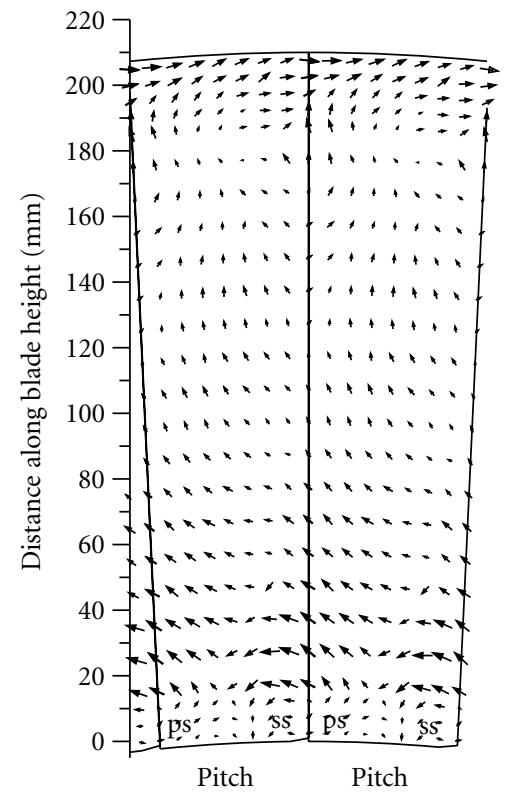

(a) Experimental

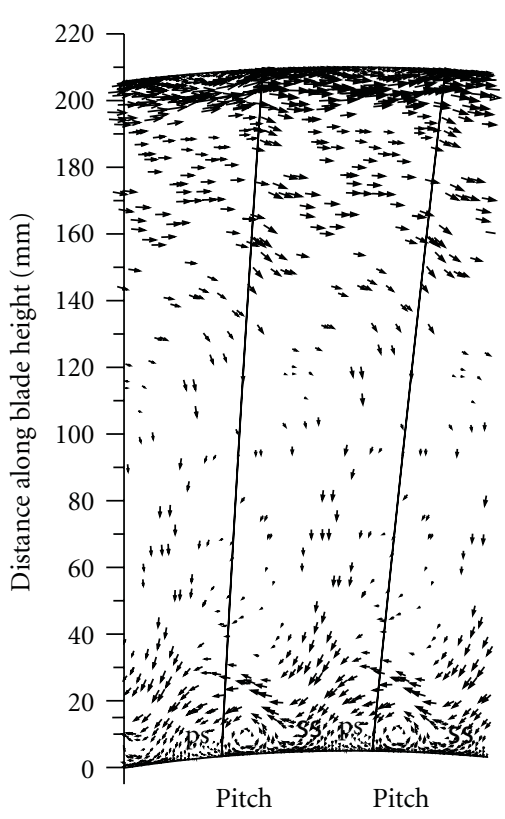

(b) Numerical

FIGURE 24: Secondary flow predicted downstream of the cascade.

\section{Conclusions}

This study introduced experimental measurements using annular sector cascade verified by numerical calculations for the effect of the radial pressure gradient on the threedimensional flow in turbine cascades. The results presented here showed that the numerical calculations and the experimental measurements provided the same flow features. The main conclusions obtained from the present study are as follows.

(a) The annular sector cascade with five blades provided reasonable results and could be used instead of fully annular cascade to reduce experiment cost and to obtain laboratory measurements for large scale blade profiles. Reducing the annular cascade to a sector rather than a full annulus reduces the required mass flow rate considerably whilst maximizing the size of the test object.

(b) The secondary flow near the hub is stronger than that near the shroud. For the blade aspect ratio considered in this study, the secondary flow was found to move across blade mid-span.

(c) The measured and the calculated total pressure loss coefficient indicated that the losses were concentrated near the hub, and therefore attempts to reduce the secondary loss should emphasize the losses near the hub.

(d) The increase in pressure difference between the blade pressure and suction surfaces with blade height increases flow deflection through blade height. Therefore, the flow is underturned near the hub and overturned near the shroud.
The radial pressure gradient would also affect the case with tip clearance gap, and the leakage flow induced from the blade pressure surface to the blade suction surface will be different from that predicted in linear cascade. In addition, the interaction between the passage vortex and the tip leakage vortex would be different from that obtained in linear cascades. Therefore, it is recommended for future work to extend the present study to the case with clearance gap.

\section{Nomenclature}

$\begin{array}{ll}a: & \text { Constant } \\ C: & \text { Chord length } \\ C_{x}: & \text { Axial chord } \\ C_{P_{t}}: & \text { Total pressure loss coefficient } \\ \overline{C_{P_{t}}:} & \begin{array}{l}\text { Mass-averaged total pressure loss } \\ \text { Coefficient }\end{array} \\ F_{1}, F_{2}: & \text { Functions } \\ h: & \text { Blade span } \\ k: & \text { Turbulent kinetic energy } \\ k_{\alpha}, k_{\beta}, & k_{t}, k_{s}: \quad \text { Probe calibration coefficients } \\ p_{1}, p_{2}, p_{3}, p_{4}, p_{5}: \text { Pressures obtained by five-hole probe } \\ P: & \text { Static pressure } \\ P_{t}: & \text { Total pressure } \\ \bar{p}: & \text { Mean pressure } \\ P_{\omega}: & \text { Production of } \omega \\ S: & \text { Blade spacing; } \\ u^{\prime}: & \text { Fluctuating velocity } \\ \bar{u}: & \text { Mean velocity } \\ \bar{V}: & \text { Mass-averaged velocity } \\ x_{i}: & \text { Coordinate in ith direction } \\ x: & \text { Distance through axial chord } \\ z: & \text { Distance along blade span. }\end{array}$




\section{Greek Symbols}

$\begin{array}{ll}\alpha: & \text { Pitch angle } \\ \beta: & \text { Yaw angle } \\ \beta_{1}: & \text { Inlet blade angle } \\ \beta_{2}: & \text { Exit blade angle } \\ \beta_{2}^{\prime}: & \text { Exit flow angle } \\ \delta: & \text { Deviation angle } \\ \beta^{\prime}, \gamma^{\prime}: & \text { Model coefficients } \\ \beta^{*}: & \text { Model constant } \\ \delta_{i j}: & \text { Kronecker second-order tensor } \\ \mu: & \text { Molecular viscosity } \\ \mu_{t}: & \text { Turbulent eddy viscosity } \\ \omega: & \text { Specific turbulent dissipation rate } \\ \Omega: & \text { Absolute value of vorticity } \\ \rho: & \text { Fluid density } \\ \sigma_{k}, \sigma_{\omega}: & \text { Model coefficients. }\end{array}$

\section{Subscripts}

1: inlet

2: exit

$t$ : tip or turbulent

$r$ : root.

\section{References}

[1] L. S. Langston, M. L. Nice, and R. M. Hooper, "Threedimensional flow within a turbine cascade passage," Journal of Engineering for Power-Transactions of the ASME, vol. 99, no. 1, pp. 21-28, 1977.

[2] C. H. Sieverding, "Recent progress in the understanding of basic aspects of secondary flows in turbine blade passages," Journal of Engineering for Gas Turbines and Power, vol. 107, no. 2, pp. 248-257, 1985.

[3] L. S. Langston, "Cross flows in a turbine cascade passage," Journal of Engineering for Power, Transactions of ASME, vol. 102, pp. 866-874, 1980.

[4] J. Moore and R. Y. Adhye, "Secondary flows and losses downstream of a turbine cascade," Journal of Engineering for Gas Turbines and Power, vol. 107, no. 4, pp. 961-968, 1985.

[5] A. Perdichizzi and V. Dossena, "Incidence angle and pitchchord effects on secondary flows downstream of a turbine cascade," Journal of Turbomachinery, vol. 115, no. 3, pp. 383$391,1993$.

[6] A. F. Slitenko and Y.M. Jukov, "Experimental investigation of three-dimensional secondary flow on the endwall of a blade channel in an axial turbine cascade," in Proceedings of the Turbine Technical Conference \& Exposition, Munich, Germany, May 2000.

[7] M. W. Benner, S. A. Sjolander, and S. H. Moustapha, "Measurements of secondary flows downstream of a turbine cascade at off-design incidence," in Proceedings of the Turbine Technical Conference \& Exposition, Vienna, Austria, June 2004.

[8] H. El-Batsh and M. Bassily Hanna, "Numerical and experimental prediction of secondary flow through a rectilinear cascade for different aspect ratios," in Proceedings of the International Mechanical Engineering Conference (IMEC '04), Kuwait, December 2004.
[9] H. El-Batsh and M. Bassily Hanna, "An investigation on the effect of endwall movement on the tip clearance loss using annular turbine cascade," International Journal of Rotating Machinery, vol. 2011, Article ID 489150, 11 pages, 2011.

[10] T. Matsunuma, "Effects of Reynolds number and free-stream turbulence on turbine tip clearance flow," in Proceedings of the Gas Turbie Technology: Focus for the Future, pp. 389-401, June 2005.

[11] C. Hirsch, "Advance methods for cascade testing," Advisory Group for Aerospace Research and Development AGARDAG-328, 1993.

[12] D. M. Vogt and T. H. Fransson, "A new turbine cascade for aeromechanical testing," in Proceedings of the 16th Symposium on Measuring Techniques in Transonic and Supersonic Flow in Cascades and Turbomachines, Cambridgem, UK, September 2002.

[13] T. Povey, T. V. Jones, and M. L. G. Oldfield, "On a novel annular sector cascade technique," in Proceedings of the Turbine Technical Conference and Exposition, pp. 1369-1380, June 2004.

[14] J. L. Gilarranz, A. J. Ranz, J. A. Kopko, and J. M. Sorokes, "On the use five-hole probes in the testing of industrial centrifugal compressors," Journal of Turbomachinery, vol. 127, no. 1, pp. 91-106, 2005.

[15] G. Ingram and D. Gregory-Smith, "An automated instrumentation system for flow and loss measurements in a cascade," Flow Measurement and Instrumentation, vol. 17, no. 1, pp. 23$28,2006$.

[16] D. C. Wilcox, Turbulence Modeling for CFD, DCW Industries, La Canada, Calif, USA, 1993.

[17] J. E. Bardina, P. G. Huang, and T. J. Coakley, "Turbulence modeling validation, testing and development," NASA Technical Memorandum 110446, 1997.

[18] B. M. Holley, S. Becz, and L. S. Langston, "Measurement and calculation of turbine cascade endwall pressure and shear stress," Journal of Turbomachinery, vol. 128, no. 2, pp. 232-239, 2006.

[19] F. R. Menter, "Two-equation eddy-viscosity turbulence models for engineering applications," AIAA Journal, vol. 32, no. 8, pp. 1598-1605, 1994.

[20] T. Hildebrandt and L. Fottner, "A numerical study of the influence of grid refinement and turbulence modeling on the flow field inside a highly loaded turbine cascade," Journal of Turbomachinery, vol. 121, no. 4, pp. 709-716, 1999.

[21] M. Casey and T. Wintergerste, ERCOFTAC special interest group on quality and trust in industrial CFD, Best Practices Guidelines, 2000. 

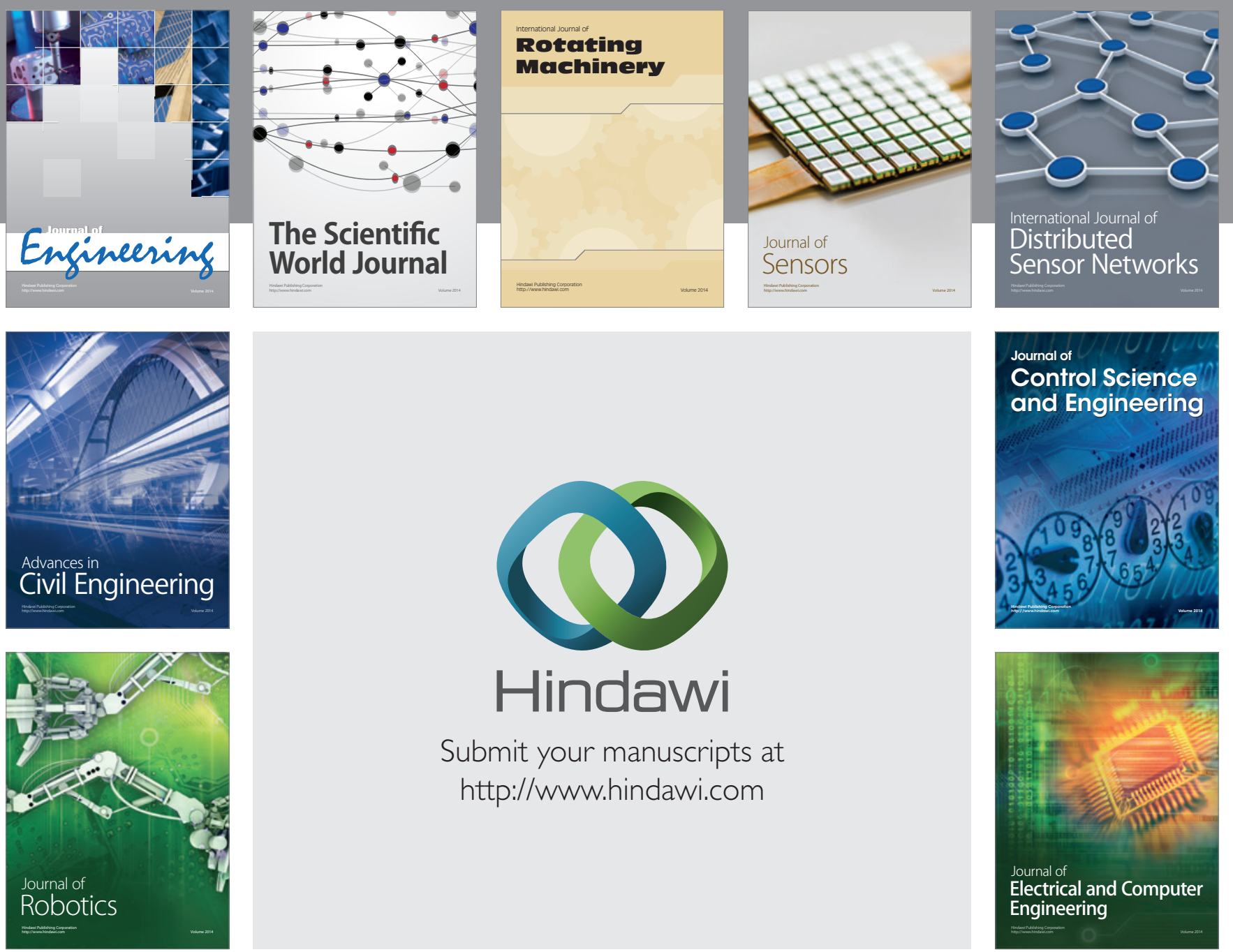

Submit your manuscripts at

http://www.hindawi.com
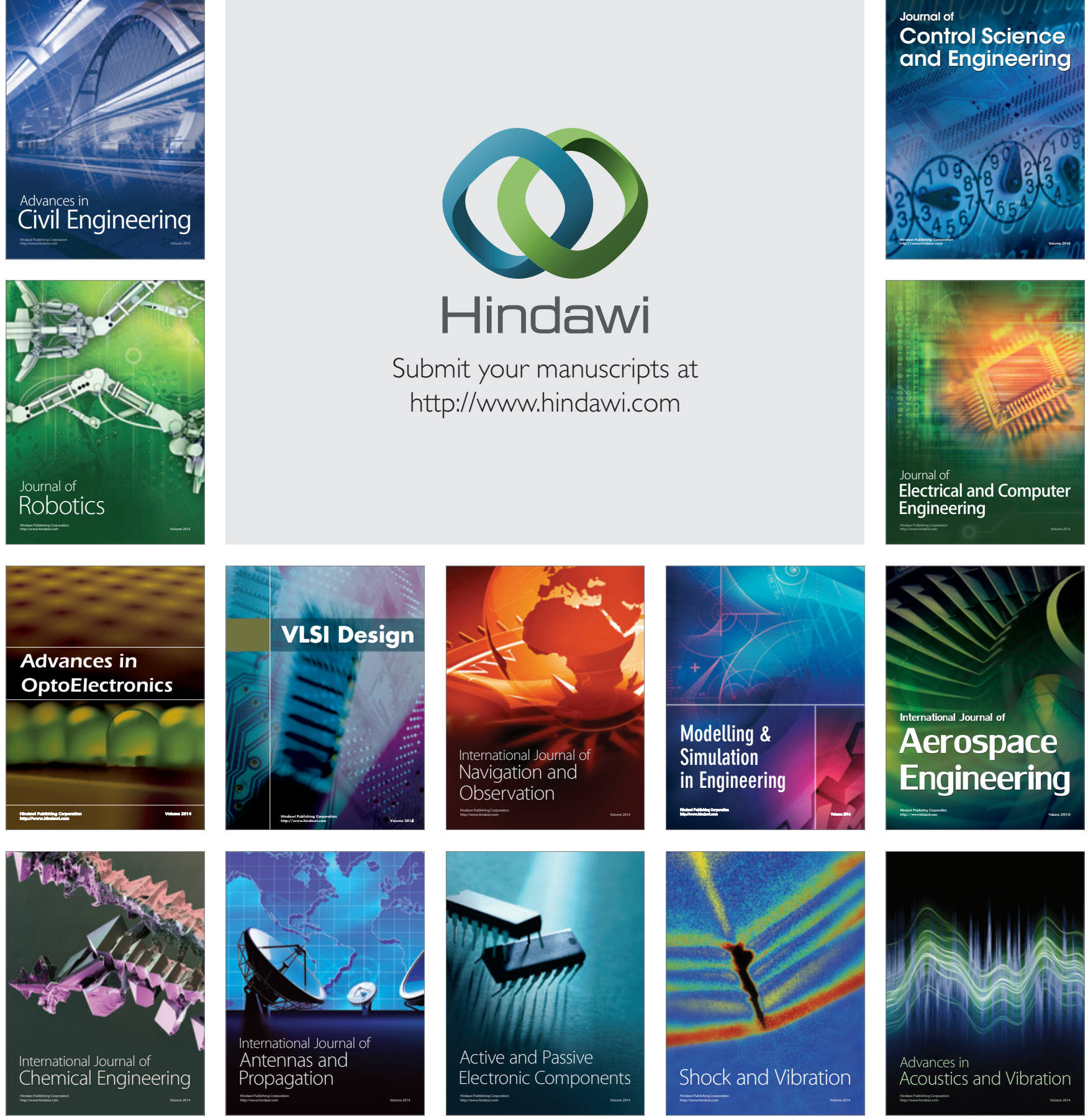